\title{
The Effectiveness of 12-Week Greek Traditional Dances Training for Improving Postmenopausal Women Cardiorespiratory Fitness
}

\author{
${ }^{1}$ Fotios Mavrovouniotis, ${ }^{2}$ Efthalia Kontaxi, ${ }^{3}$ Eirini Argiriadou, \\ ${ }^{4}$ Asterios Deligiannis \\ School of Physical Education and Sport Sciences, Aristotle University of Thessaloniki, 54124-GREECE \\ mavrov@phed.auth.gr
}

\begin{abstract}
The purpose of the present study was to examine the effectiveness of 12-week Greek traditional dances training for improving postmenopausal women cardiorespiratory fitness. Twenty three postmenopausal women, aged 55-68 years, were, randomly, chosen and separated to an experimental group $(n=13)$ that attended a 12-week Greek traditional dances program at a frequency of three sessions per week, 50 minutes each, and a control group $(n=10)$ that continued their daily habits for the same period. Cardiorespiratory fitness was assessed by the six-minute walking test $(6 \mathrm{MWT})$ and resting heart rate (HR). For data analysis, the non-parametric test Wilcoxon and Mann-Whitney $U$ test, and Pearson correlation of the SPSS ver. 20.0 for windows was used. No significant difference in the baseline measures was detected between the two groups. After the intervention program it was found out a significant decrease in resting $H R(z=-2.50, p<0.05)$ for the experimental group, but an increase for the control group. A significant increase in distance travelled during the 6MWT ( $z=-3.18$, $\mathrm{p}<0.01)$ was observed in the experimental group, while the control group presented a significant decrease $(z=-$ $2.49, p<0.05)$. The results provide evidence for the effectiveness of Greek traditional dances programs in improving the cardiorespiratory fitness of postmenopausal women.
\end{abstract}

Key-words: elderly, aerobic exercise, cardiopulmonary performance, functional capacity.

Academic Discipline / Sub Discipline: Physical Education, Fitness

Subject Classification: Physical Education

Type: Quasi-Experimental

Date of Publication: $30-05-2018$

DOI: $10.24297 / j s s r . v 12 \mathrm{i} 2.7447$

ISSN: 2321-1091

Volume: 12 Issue: 2

Journal: Journal of Social Science Research

Publisher: CIRWORLD

Website: https://cirworld.com

This work is licensed under a Creative Commons Attribution 4.0 International License. 


\section{Introduction}

Menopause, the permanent cessation of menstruation due to complete depletion of the follicle pool, is postulated twelve months after the last menstrual period (Vryonidou, \& Klonaris, 2010). The period of transition to menopause lasts from 2 to 9 years and is characterized by a multitude of endocrinological, biological, clinical and psychological changes, which are partly due to loss of number and function of the follicles, while others are the result of the normal aging process. Menopause usually occurs in the late 40 s to early 50 s (Diakoumakou, $\&$ Diakoumakos, 2010; Edwards, \& Li, 2000; North American Menopause Society, 2010; Palacios, 2010).

Women, after menopause, experience many pathophysiological changes. Estrogen reduction predisposes to the occurrence of atherogenic dyslipidemia, which is one of the most important risk factors for the development of cardiovascular diseases in the female population and contributes by $25-30 \%$ to the increase of cardiovascular risk in postmenopausal women (American Heart Association, 2011; Diakoumakou, \& Diakoumakos, 2010; Elisaf et al., 2014; Knopp, 2002; Kolovou, \& Bilianou, 2008).

More specifically, a pathological lipidemic profile, characterized by increased LDL-C, or/and combined hyperlipidemia with increased LDL-C, elevated TG, low HDL-C, is recorded in the peri- and postmenopausal women population. In addition to atherogenic lipidemia, the effects of hypo-estrogenemia, during menopause, also include an increase in body weight, body mass index, intra-abdominal fat and waist circumference. Moreover, there is an increase in blood pressure, glucose metabolism disorders and resistance to insulin action. Estrogens play an important role in local and systematic vasodilation through mechanisms dependent and nondependent from the endothelium. During menopause and age progress, there is also a decrease in elasticity in large arteries (such as carotid, aorta) which is an independent cardiovascular risk factor. Changes in the levels of coagulation factors are also observed. The installation of an over-coagulated condition is associated with an increased risk for a cardiovascular occurrence (Edwards, \& Li, 2000; Mavroudi et al., 2010; Zapantis, \& Santoro, 2003).

Therefore, it can be said that cardiac function tends to decline after menopause. Thus, a fifty-year-old woman has a $50 \%$ chance of developing cardiovascular disease and a $31 \%$ chance of losing her life, with the average death rate from coronary artery disease at 74 years (Rousseau, 1998). It is worth noting that cardiovascular risk in women is equated with that of men about a decade after menopause (American Heart Association, 2014; Haddock et al., 2000). In addition, the lung capacity of menopausal women older than 40 years normally decreases 30-50 mL annually (Barr et al., 2004; Real et al., 2008).

With regard to hormone replacement therapy, the results are contradictory and recent guidelines indicate that it should not be used for the primary or secondary prevention of cardiovascular disease (American Heart Association, 2011). In addition, estrogen replacement therapy may increase the incidence of newly diagnosed asthma and chronic obstructive airway diseases (Barr et al., 2004). Thus, it is necessary to consider the potential impact of non-pharmaceutical interventions that can help in this direction. In this context, regular aerobic exercise and the induced improvements on cardiopulmonary resistance are consistently among the most effective means of preventing cardiovascular disease in postmenopausal women (American Heart Association, 2011; Blumenthal et al. 1991; Haddock et al., 2000).

It is worth noting that a gradual inverse relationship between women's physical activity and the risk of cardiovascular death has been demonstrated (Oguma, \& Shinoda-Tagawa, 2004). Thus, a physically active middle-aged woman runs half the risk of dying from a cardiovascular event compared to a woman of the same age who does less than one hour exercise per week ( $\mathrm{Hu}$, et al., 2004). It can therefore be said that naturally active postmenopausal women over 45 years of age have a more favorable profile with regard to risk factors for cardiovascular disease including blood lipid levels (Mensink, 1996; Mora et al. 2007). In addition, from reviewing the literature it has been clear that the participation of postmenopausal women in aerobic exercise programs 
leads to an increase in cardiorespiratory resistance associated with the improvement of their lipidemic profile (Green et al., 2004; Haddock et al., 1998; Hagner et al., 2009; Karolkiewizc et al., 2009; Kelley et al., 2004).

However, although the effects of aerobic exercise on people's cardiorespiratory fitness have been studied (Blumenthal et al., 1991; Halverstadt et al., 2007; Korshoj et al., 2016; Lin, et al., 2015; Roussel et al., 2009) the effects of dance, and more specifically the effects of Greek traditional dances on women cardiorespiratory fitness have not been studied yet. Therefore the purpose of the present study is to examine the effects of a 12week Greek traditional dances program on the cardiorespiratory fitness of postmenopausal women.

\section{Materials and Methods}

\section{Sample}

From the lists of the members kept in Adult Club of Lemesos City of Cyprus, thirty eight (38) members, all women, that fulfilled the inclusion criteria, that is age 55-68 years old and no participation in any group or individual exercise, physical activity or dancing program for the past six months, were randomly chosen. Subsequently, a communication/invitation to each chosen member, in regard to the research was made. After that, a total of thirty six women volunteered to participate in the research.

A written informed consent for the participation in the research was obtained from each woman. All the women, before the beginning of the research, underwent medical control so that it could be certified that they do not suffer from any cardiovascular or other disease and, also, that they do not take any medication. Additionally, they answered a questionnaire about any health problems, while a research assistant was present in order to give any essential clarifications if there were any questions. Seven women who were found to fulfil the exclusion criteria, that is menstruation the past 12 months, health problems, or/and medication that could affect the results, or/and participation in any group or individual exercise, physical activity or dancing program for the past six months, or/and cigarette smoking, were excluded from the research. Finally, twenty nine (29) healthy postmenopausal women participated in the research. Subjects' age ranged from 55 to 68 years $(M=60.09, S D=4.089)$. The subjects were, then, separated randomly to an experimental (group $A)(n=15)$ and a control group (group B) $(n=14)$. From them, at the end of the research, regarding the experimental group, the data of two women were excluded because, due to illnesses or/and other reasons, they missed more than three Greek traditional dances bouts. In addition, regarding the control group, the data of four women were excluded because, due to illnesses or/and other reasons, they missed the final measurements. Finally, thirteen healthy postmenopausal women of experimental group and ten healthy postmenopausal women of control group were participated in the study and were studied for the purposes of the research.

\section{Procedure}

An approval for conducting the research was given from the committee of the Adult Club of Lemesos City of Cyprus, after the aim and the treaties of the research were described. Procedures were in agreement with the ethical standards of the Declaration of Helsinki of the World Medical Association (2000).

All the subjects came to the Club, in scheduled afternoon hours. Before the beginning of the research, a description of general requirements was given and the aim of the research was also described to the women without any briefing relative to previous research findings. The six minute walking test (6MWT) for cardiopulmonary performance measure (was also presented and the instructions were explained. Then, was announced to the participants women in which group they randomly separated.

As for experimental group, particular emphasis was given on the need for regular participation in the program. It was, also, noted that a woman who missed more than three Greek traditional dances bouts ( 3 absences out of 36 sessions), for any reason, would be excluded from the research. As for control group, particular emphasis was given on the need for participation in the two measurements, before and after the 12-week period. It was, 
also, noted that a woman who missed one of the two measurements, for any reason, would be excluded from the research.

Moreover, the subjects of the experimental group received one-hour preparatory session, on methods of heart rate $(\mathrm{HR})$ measurement and procedural details. During this session, the subjects were trained to self-measure their HR using the palpation method. The wrist location (radial artery) was chosen. The subjects learned to place their index and middle fingers together on the wrist of the other hand, about $1 / 2$ inch on the inside of the joint, in line with the index finger, so they could feel the pulse. After a practice in taking their HR, the accuracy of their HR measurement was checked by pre-trained assistants.

Afterwards, the women of the experimental group participated in a 12-week group program of Greek traditional dances performance at a frequency of 3 training sessions per week, for 50 min each session, which has been considered an optimal training as for the duration and the frequency (Adiputra et al., 1996; Blackman et al., 1988). Each Greek traditional dances session included the warming up for 5 min which contained dances of low to moderate intensity, the main part for 40 min which contained dances of moderate to high intensity and the cool-down for 5 min which contained dances of low intensity. Especially for the main part, the dances intensity ranged from moderate to high, so that the HR ranges from $60-75 \%$ of the maximum HR (HRmax) (Karvonen, 1957). In addition, there were frequent rhythm alternations so that the subjects could keep dancing continuously throughout each dance session. Essential breaks of approximately $15-20 \mathrm{sec}$ in between dances in order to change dance were made. The duration of each dance was about 2.5 to $3.5 \mathrm{~min}$.

The performed Greek traditional dances were from different areas of Greece. The program was designed according to certain basic dances, while the purpose was to enrich the program with a variety of dances, regarding the rhythm, the kinetic repertoire and the style. In order to begin dancing, women were holding each other using a variety of handholds, creating a hemi-cycle. The dances that were performed included a variety of simple kinetic patterns with music accompaniment. More specifically, during the dances, they performed small, moderate or long steps, slow, medium, fast or very fast steps, movements forward, back, right or left, one or more turns, rebounds on one or both legs, blows to the ground with one or both feet, stops on one or both legs. The movements of the lower limbs were in co-ordination with specific movements of the upper limbs such as forward, backward, upward movements, etc, always with music accompaniment (Table 1).

Table 1. Performed Greek traditional dances during the program

\begin{tabular}{llcc}
\hline & Traditional dance & Musicmeter & Handhold \\
\hline Warm-up & Berati of Thessaly & $7 / 8$ & Free-without handhold \\
& Syrtos & $7 / 8$ & W-shape \\
\hline & Tsamikos & $3 / 4$ & W-shape \\
& Tasia & $2 / 4$ & Free-without handhold \\
& Fyssouni & $9 / 8$ & Shoulder grip \\
& Chromatista Tsourapia & $9 / 8$ & Simple-arms down \\
& Trechatos & $7 / 16$ & W-shape \& Simple-arms down \\
& Raiko & $7 / 16$ & Simple-arms down \\
Main Part & Zonaradikos & $6 / 8$ & W-shape
\end{tabular}




\begin{tabular}{lcc}
\hline Sfarlis & $9 / 8$ & Simple-arms down \\
Baidouska & $5 / 8$ & Simple-arms up \& down \\
Xesyrtos & $2 / 4$ & W-shape \\
Bogdanos & $7 / 8$ & W-shape \& up-down \\
Syrtos Sygathistos & $9 / 8$ & W-shape \& up-down \\
Tremouliastos & $2 / 4$ & W-shape \\
Tik & $5 / 8$ & W-shape \& up-down \\
Syrtos Chaniotis & $2 / 4$ & W-shape \\
Balos & $2 / 4$ & W-shape \\
\hline Syrto sta tria & $4 / 4$ & W-shape \\
Pogonisios & $4 / 4$ & W-shape
\end{tabular}

At the same 12-week period, the women of the control group were asked to continue their daily lives without any changes as it was recorded at the beginning of the research, concerning medication that could affect the results, or/and participation in any group or individual exercise, physical activity or dancing program. Measurements and each session of Greek traditional dances were conducted by a single, female investigator, teacher of physical education with extensive practical experience in Greek traditional dances.

\section{Measurements}

Anthropomorphological Measurements. Measurements of body mass and height were taken place. Body mass was measured using a Microlife WS80 electronic scale with a precision of $0.1 \mathrm{~kg}$. Body height was measured with a precision of $0.5 \mathrm{~cm}$ using a Seca 216 height measuring. In addition, Body Mass Index (BMI) was estimated (body mass/height ${ }^{2}$ ).

Resting Heart Rate. Resting HR was measured from the same physical education teacher, in the morning, between 8.00 and 10.00 before noon, after 12 hours of caffeine consumption avoidance. The physical education teacher measured the subjects HR during two 10-sec periods, using the palpation method, on the wrist location (radial artery), which multiplied by 4 gave subjects' HR, beats per minute. The average of the two measurements was calculated. Two resting HR measurements were carried out, in order to evaluate the effects of the Greek traditional dances program. The initial measurement was carried out before the beginning of the 12-week Greek traditional dances program and the final measurement after the 12-week Greek traditional dances program.

Dancing Heart Rate. HR response to dancing was measured for assessing workout intensity. So, just before the first Greek traditional dances session of the program, the physical education teacher measured the subjects $\mathrm{HR}$ at rest during two 10 -sec periods. In continuity, the Greek traditional dances session began and was interrupted for a period of no longer than 1:00 to 1:30 min, during which the subjects measured their HR on the $5^{\text {th }}$ min- that is the end of the warm-up-, as well as on the $13^{\text {th }}, 26^{\text {th }}$ and $40^{\text {th }}$ min of the main part of the Greek traditional dances session. The teacher of physical education and pre-trained assistants supervised the HR measurements after averaging the two HR. The intensity of Greek traditional dances was calculated using the Karvonen formula (Karvonen et al., 1957). 
Cardiorespiratory fitness. In order to measure the cardiorespiratory fitness, the 6MWT was used. The 6MWT is an objective measure of exercise tolerance, easy to administer and more reflective of activities of daily living (American Thoracic Society, 2002; Butland et al., 1982; Solway et al., 2001). Subjects were instructed to walk back and forth along a 30-m hallway of flat and hard surface, which is delimited by two cones, as fast as possible, for $6 \mathrm{~min}$. In addition, they were urged to spin/change direction as soon as possible. Before the beginning of the test, the need for fast walking and spin / change of direction was particularly emphasized. At the end of the 6 min period, the total distance travelled was recorded.

Data analysis. For the statistical analysis the statistic packet SPSS/PC Version 20.0 for windows was used. Moreover, descriptive analysis was used, and the non-parametric Wilcoxon 2-related sample test, as well, in order to evaluate significant differences between measurements (before and after the Greek traditional dances program as for intervention group, and before and after the 12-week period as for control group). In addition, the non-parametric Mann-Whitney $U$ test was used to evaluate significant differences between the groups. Pearson correlation was also used to evaluate significant correlations between the studied variables. The level of significance was set to $p<0.05$.

\section{Results}

In Table 2 the anthropomorphological characteristics of the subjects of the experimental group pre and post the 12-week intervention, as well as of the control group pre and post the 12-week period are presented. As for the anthropomorphological characteristics, there was no significant difference between the two groups. However, weight and BMI was significantly decreased in experimental group after the participation in the 12week Greek traditional dances program $(p<0.05)$. On the contrary, no significant difference was found in the control group (Table 2).

Table 2. Sample's anthropomorphological characteristics pre and post the 12-week intervention

\begin{tabular}{|c|c|c|c|c|}
\hline & \multicolumn{2}{|c|}{ Experimental Group } & \multicolumn{2}{|c|}{ Control Group } \\
\hline Variables & Pre & Post & Pre & Post \\
\hline Age (years) & \multicolumn{2}{|c|}{$60.08 \pm 4.35$} & \multicolumn{2}{|c|}{$60.1 \pm 3.96$} \\
\hline Height $(\mathrm{cm})$ & \multicolumn{2}{|c|}{$1.58 \pm 0.04$} & \multicolumn{2}{|c|}{$1.55 \pm 0.04$} \\
\hline Weight (Kg) & $66.63 \pm 12.06$ & $65.71 \pm 12.06^{*}$ & $71.37 \pm 8.06$ & $71.65 \pm 8.60$ \\
\hline Body Mass Index & & & & \\
\hline (BMI) $\left(\mathrm{Kg} / \mathrm{m}^{2}\right)$ & $26.57 \pm 4.90$ & $26.20 \pm 4.89^{*}$ & $29.50 \pm 4.13$ & $29.64 \pm 4.49$ \\
\hline
\end{tabular}

${ }^{*} \mathrm{p}<0.05$ between initial and final measurement.

In Figure 1 the mean values of the HR of subjects at rest, at $5^{\text {th }} \min$, that is during the warming-up, as well as at $18^{\text {th }}, 31^{\text {th }}$, and $45^{\text {th }}$ min of the main part of one typical session of the Greek traditional dances program, are presented. 


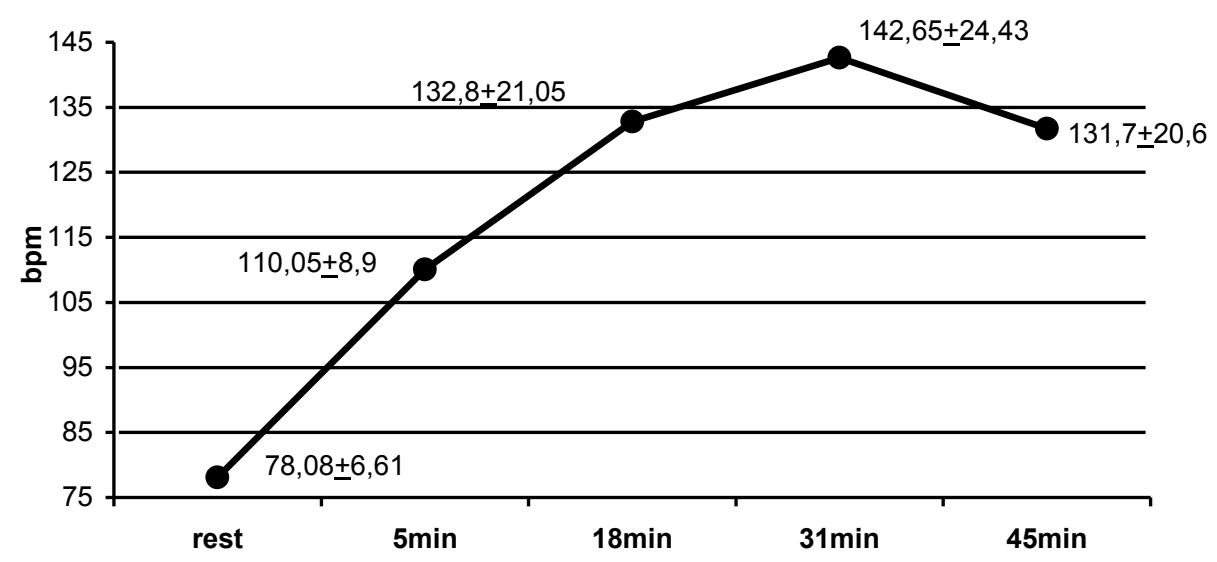

Figure 1. HR fluctuation (mean \pm SD) during the warming-up and the main part of a typical session of the Greek traditional dances program

As it is observed, while at rest the subjects presented HR $78.08 \mathrm{bpm}$ and reached the end of the main part of a typical session of Greek traditional dances program, that is after $45 \mathrm{~min}, 131.7 \mathrm{bpm}$, a HR that is within the range of training zone for the age of 60 (American College of Sports Medicine, 1991; Chase, http://plu.edu/ chasega/main.htm). This increase represents approximately $65 \%$ of HRmax (Figure 1 ).

Descriptive statistics for the resting HR assessed prior to and following the 12-week Greek traditional dances program for the experimental group and prior to and following the 12-week period for the control group, as well as the significance of any demonstrated change are shown in Figure 2 and Table 3.

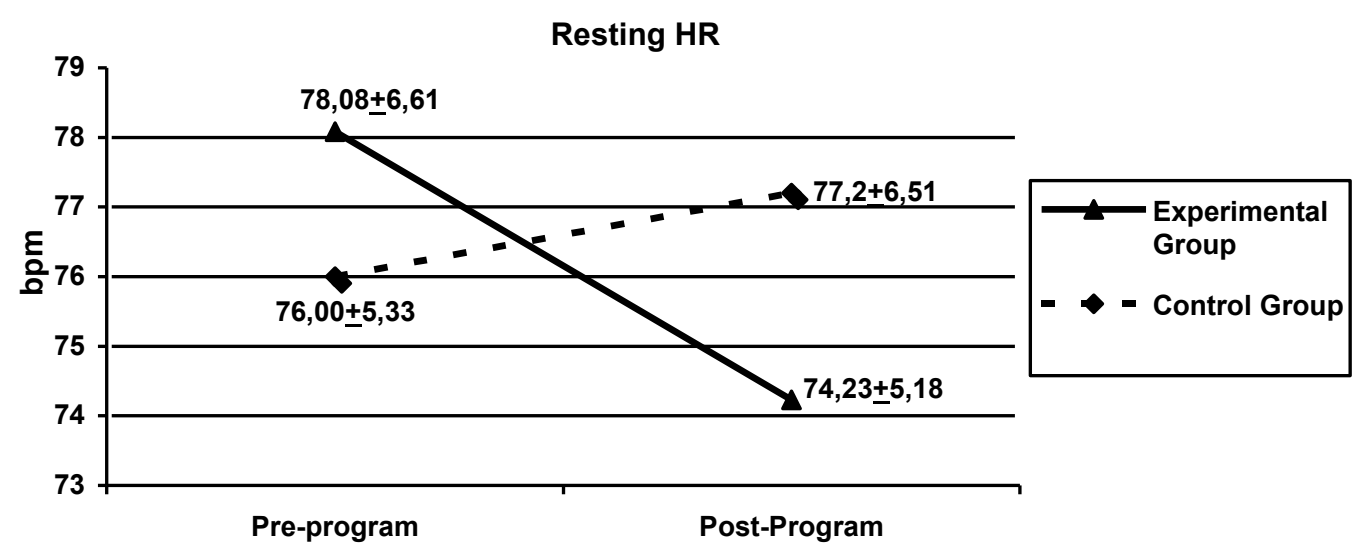

Figure 2. Resting HR (mean $\pm S D)$ for both groups

According to data processing, it was obvious that there was a significant decrease in resting HR (4.93\%) for the experimental group after the participation in the 12-week Greek traditional dances program. On the contrary, there was observed an increase in resting HR (1.55\%) for the control group after the 12-week period (Table 3).

Table 3. Descriptive data, and degree of change for resting HR

\begin{tabular}{|l|c|c|c|c|c|c|c|}
\hline \multirow{2}{*}{$\begin{array}{l}\text { Resting HR } \\
(\mathrm{bpm})\end{array}$} & \multicolumn{2}{|c|}{ Pre-program } & \multicolumn{2}{|c|}{ Post-program } & Change & & \\
\cline { 2 - 8 } & $\mathrm{M}$ & $\mathrm{SD}$ & $\mathrm{M}$ & $\mathrm{SD}$ & & $\mathbf{z}$ & $\mathbf{P}$ \\
\hline Experimental & 78.08 & 6.61 & 74.23 & 5.18 & $\downarrow$ & -2.50 & $<0.05$ \\
\hline
\end{tabular}




\begin{tabular}{|l|l|l|l|l|l|l|l|}
\hline Group & & & & & & & \\
\hline Control Group & 76.00 & 5.33 & 77.20 & 6.51 & $\uparrow$ & NS \\
\hline
\end{tabular}

From the non-parametric test Wilcoxon it was found out that resting HR of the experimental group was significantly influenced by the Greek traditional dances program $(z=-2.50, p<0.05)$. This increase reflects positive responses of the participants, postmenopausal women, in the Greek traditional dances program. As for control group, the observed increase in resting HR was not significant (Table 3). In addition, from the nonparametric Mann-Whitney $U$ test it was found out that there was no significant difference between the two groups, experimental and control, as for the measurements of resting HR before and after the 12-week intervention.

Descriptive statistics for the distance travelled during the 6MWT prior to and following the 12-week Greek traditional dances program for the experimental group and prior to and following the 12 -week period for the control group, as well as the significance of any demonstrated change are shown in Figure 3 and Table 4 .

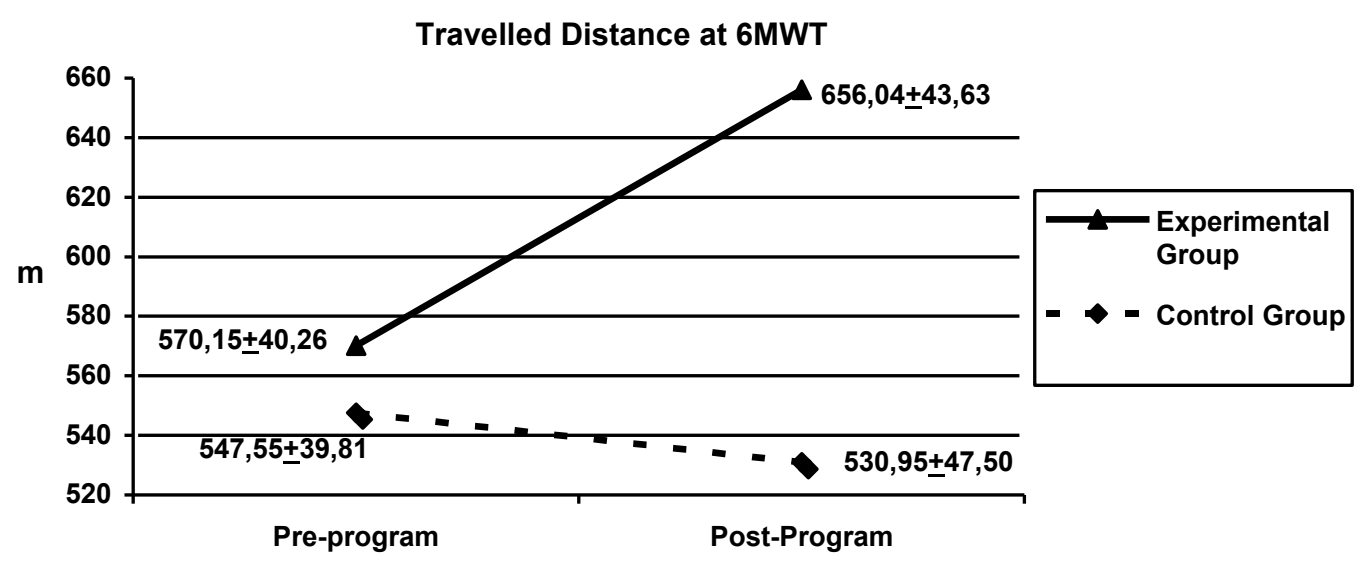

Figure 3. The distance travelled during the 6MWT for both groups

According to data processing, it was obvious that there was a significant increase in distance travelled during the 6MWT (15.06\%) for the experimental group after the participation in the 12-week Greek traditional dances program. On the contrary, there was observed a significant decrease in distance travelled during the 6MWT (3.03\%) for the control group after the 12-week period (Table 4).

Table 4. Descriptive data, and degree of change for distance travelled during the 6MWT for both groups

\begin{tabular}{|l|c|c|c|c|c|c|c|}
\hline \multirow{2}{*}{$\begin{array}{l}\text { Distance } \\
(\mathrm{m})\end{array}$} & \multicolumn{2}{|c|}{ Pre-program } & \multicolumn{2}{|c|}{ Post-program } & Change & & \\
\cline { 2 - 8 } & $\mathrm{M}$ & $\mathrm{SD}$ & $\mathrm{M}$ & $\mathrm{SD}$ & & $\mathbf{z}$ & $\mathbf{p}$ \\
\hline $\begin{array}{l}\text { Experimental } \\
\text { Group }\end{array}$ & 570.15 & 40.26 & 656.04 & $43.63^{* * \wedge}$ & $\uparrow$ & -3.18 & $<0.01$ \\
\hline Control Group & 547.55 & 39.81 & 530.95 & $47.50^{*}$ & $\downarrow$ & -2.49 & $<0.05$ \\
\hline
\end{tabular}

${ }^{* *} p<0.01$ between initial and final measurement. ${ }^{*} p<0.05$ between initial and final measurement.

$\wedge p<0.001$ between groups at final measurement. 
From the non-parametric test Wilcoxon it was found out that the cardiorespiratory fitness of the experimental group, as it was measured by the distance travelled during the 6MWT, was significantly influenced by the Greek traditional dances program $(z=-3.18, p<0.01)$. This increase reflects positive responses of the participants, postmenopausal women, in the Greek traditional dances program. As for the control group, the observed decrease in distance travelled during the 6MWT was, also, significant $(z=-2.49, p<0.05)$. In addition, from the non-parametric Mann-Whitney $U$ test it was found out that there was a significant difference between the two groups, experimental and control, as for the measurement of the distance travelled during the 6MWT after the 12-week intervention $(z=-3.91, p<0.001)$, with the experimental group travelled significantly more meters distance during the six minutes (23.56\%) compared with the control group (Table 4).

Correlational analyses between the various measures taken post-program were then conducted to determine the degree to which such measures were or were not associated (Table 5).

Table 5. Correlations between studied variables after the 12-week intervention

\begin{tabular}{|c|c|c|c|c|}
\hline & Height & Weight & BMI & 6MWT \\
\hline Height & 1.00 & & & \\
\hline Weight & -.10 & 1.00 & & \\
\hline BMI & $-.40^{\star}$ & $.95^{\star \star *}$ & 1.00 & \\
\hline 6MWT & $.37^{+}$ & $-.48^{*}$ & $-.59 * *$ & 1.00 \\
\hline
\end{tabular}

From the results presented in Table 5, it appears that the distance travelled during the 6MWT correlated negatively with the measures of weight and BMI, indicating that as much weight and BMI increase, so much the distance travelled during 6MWT will decrease. Moreover, a trend for positive correlation $(r=0.37, p=0.08)$ presented between height and the 6MWT score.

\section{Discussion}

According to WHO (2011), adults aged 18-64 should do at least 150 minutes of moderate-intensity aerobic activity throughout the week or at least 75 minutes of high intensity aerobic physical activity or an equivalent combination of moderate and intense activity. Neverthless, it appears that WHO guidelines are not met, as participation rates in physical activity are small. Globally, about $28 \%$ of men and $34 \%$ of women are insufficiently active (Hallal et al., 2012; Haskell et al., 2009; WHO, 2010). More specifically, in USA and Eastern Mediterranean almost the $50 \%$ of women are inactive. In agreement, in Europe, more than one quarter of adults are not sufficiently active (Hallal et al., 2012; International Sport and Culture Association, 2015; WHO, 2014), while in Greece sedentary life rates were over $60 \%$ (Makrilakis et al., 2004). It is worth to be mentioned that inactivity is higher in women than in men and increases with age, as well as in countries with high income, where almost one in two women are not sufficiently physically active (Hallal et al., 2012; International Sport and Culture Association, 2015; WHO, 2014). In addition, physical inactivity is commonly observed among individuals aged $\geq 60$ years. Identified barriers to sedentary older adults beginning activity include low self-efficacy, preexisting medical conditions, physical limitations, time constraints, and culture (Hwang, \& Braun, 2015).

In order to achieve the regular participation of middle-aged and elderly people in exercise programs, the exercise/activity must be well-accepted and pleasant for the participants (Grant et al., 2002). Extra attention should be paid to older participants in physical activities, especially older women, because they are in the period of menopause (Asikainen et al., 2003). In addition, exercise programs that can resonate mostly in adults 
over 50 years are more moderate in intensity, simply and conveniently on participation, relatively low cost, uncompetitive, and especially for women, covering social interaction needs (King, 2001). In this direction, dance can help, as it could be an option that can make physical activity more interesting and attractive (Arts council England, 2006; Judge, 2003), and so can be adjusted to fit a target populations age, physical limitations, and culture (Hwang, \& Braun, 2015). Moreover, dance sport is a form of aerobic exercise that does not require special equipment and can be performed anywhere regardless of season or weather. Dancing is an ideal exercise to relieve tension and pressure, and it is an enjoyable social activity that improves fitness levels (SeHong et al., 2011).

With regard to Greek traditional dances, it has been proven that Greek traditional dances as a form of exercise with music accompaniment are considered a pleasant exercise type, since participants in Greek traditional dances declare that enjoy highly doing Greek traditional dances. Besides it was concluded that the enjoyment constitutes a significant factor that could affect the subjective health of the participants in the Greek traditional dances (Argiriadou et al., 2013). Moreover, middle-aged people who participate in a Greek traditional dances program declared that the participation in Greek traditional dances offered them joy, well-being and calmness (33.30\%), many effects on their bodies (33.30\%), as well as sociability-participation in a group (16.70\%) and activity increase (16.70\%) (Argiriadou et al., 2017). In addition, the elderly participants in circular dances, such as Greek traditional dances, recognize the importance of exercise for health and disease prevention (Bird et al., 2009; Eyigor et al., 2009; Hui et al., 2009; Mavrovouniotis et al., 2016; Mavrovouniotis, \& Argiriadou, 2008; Papaioannou et al., 2009/2010). Thus, Greek traditional dances may be an alternative form of physical activity aimed at the activation of postmenopausal women and the fight against sedentary lifestyle that is one of the causes of the prevalence of cardiovascular diseases in women (American Heart Association, 2011; Arts council England, 2006).

It is worth to be mentioned that Greek traditional dances are not just a cultural element of Greece, but, also, an aerobic exercise, as they meet the criteria of American College of Sports Medicine (1995) that is, they are performed in a HR training zone of approximately $60 \%$ to $90 \%$ of $\mathrm{HR}_{\max }$ (American College of Sports Medicine, 1995). So, Argiriadou (2013) found that Greek traditional dances performed in a HR training zone of approximately $78-79 \%$ of $\mathrm{HR}_{\max }$ as for middle aged and elderly participants. As for old participants, Mavrovouniotis et al. (2010) found that they performed in a HR training zone of approximately $63 \%$ of $H R_{\max }$. In agreement, from the present results, it was found that the performed Greek traditional dances sessions of the 12 -week program was moderate in intensity, as they performed in a HR training zone of approximately $65 \%$ of $H R_{\text {max }}$. Thus, Greek traditional dances, indeed, are an aerobic exercise mode. Besides, high intensity exercise is not practical for the majority of older women and is associated with more musculoskeletal injuries and less participation. Moreover, high intensity is not considered necessary for health benefits (King et al., 1995; Lindheim et al., 1994).

In addition, Greek traditional dances programs may enhance the cardiorespiratory fitness. More specifically, from the results of the present study it appeared that the participating postmenopausal women in the program of the Greek traditional dances increased significantly the distance travelled in the 6MWT. In detail, after the Greek traditional dances program there was observed an average increase in the travelled distance of 86 meters, that is an increase of $15 \%$ compared with the measurement before the program. It is worth to be mentioned that this specific field test evaluates the global and integrated responses of all the systems involved during exercise, including the pulmonary and cardiovascular systems, systemic circulation, peripheral circulation, blood, neuromuscular units, and muscle metabolism. The self-paced 6MWT assesses the submaximal level of functional capacity (American Thoracic society, 2002). Since, the 6MWT indicates the cardiovascular endurance of the heart and blood vessels (Janyacharoen et al., 2015; Swisher, \& Goldfarb, 1998), in individuals, adults and elderly, 40-94 years old (Enright, \& Sherrill, 1998; Rikli, \& Jones, 2012), it could be said that following the 12-week program of Greek traditional dances, the cardiovascular endurance of the participating postmenopausal women increased significantly. 
On the contrary, the control group decreased significantly the travelled distance by $3 \%$. In addition, although there was no significant difference between the initial measurements of the two groups, experimental and control, as for the travelled distance in $6 \mathrm{MWT}$, the difference between the two groups at the final measurements after the 12 -week period, by $23.56 \%$, was significant. Thus, it can be said that Greek traditional dances program may improve cardiorespiratory fitness of postmenopausal women.

The importance of increasing cardiorespiratory fitness of postmenopausal women of the present sample is very high since, from one side the cardiorespiratory fitness is a strong and independent prognostic indicator for possible occurrence of coronary heart disease (Thompson et al., 2003), but on the other side high cardiorespiratory fitness level is associated with lower mortality in women (Farrel et al., 2002). In addition, the improvement of cardiorespiratory fitness seems to result in easier execution of daily activities and thereby in the improvement of the quality of life (Lindheim et al., 1994), as well as of the functional capacity of postmenopausal women.

This may happen in the present study, once, indeed, the travelled distance on 6MWT may better reflect the functional exercise level for daily physical activities (American Thoracic society, 2002). Here lies the value of the present Greek traditional dances program, since the aim of the exercise programs for the elderly is to increase their reserve capacities in order to be able to perform daily life activities. Besides, the definition of health for the older people is the maintenance of functional independence until death (King et al., 2002).

With regard to older people, there is no consensus on the appropriate quantity, quality, or intensity of exercise that is necessary to promote better health and function in this population (Vincent et al., 2002). However, it has been proposed that optimal training should last 50-60 min at a time, at a frequency of three times per week for 12 weeks (Adiputra et al., 1996; Blackman et al., 1988) that is the duration and frequency of the present dancing program.

In addition, Denazi et al. (2013) who, also, applied Greek traditional dances found that the women, 60-78 years old, who participated in a 14-week Greek traditional dances program, at a frequency of 3 training sessions per week, for 50 minutes each session, improved significantly the aerobic endurance as measured by the 6MWT, while no significant improvement was observed in the control group. More specifically, the travelled distance by the participant women in the 14-week Greek traditional dances program was increased from $397.211 \pm 51.31 \mathrm{~m}$ to $498.684 \pm 77.76 \mathrm{~m}$.

In similar findings led a Thai dance program applied 3 times per week for 6 weeks in menopausal women aged $54.6 \pm 6.4$ years, while the control group received general health guidance (Janyacharoen et al., 2015). Each Thai dance session was divided into a 10-minute warm-up and muscle stretching, 40 minutes of Thai dance, and 10 minutes of cool-down stretching. The standard Thai dance performed consisted of traditional songs and postures that included arm raising, lowering, bending, stretching, alternately raising the right and left arms, raising and lowering the legs, stretching the knees, standing on the toes and feet, and turning around. After the 6 -week Thai dance program, the mean 6MWT distance travelled in Thai dance group was significantly higher $(285.4 \pm 38.8 \mathrm{~m})$ than that at baseline $(254.8 \pm 37.3 \mathrm{~m})$ and the control group at baseline $(252.8 \pm 40.3 \mathrm{~m})$ as well as the control group post-intervention $(247.0 \pm 50.2 \mathrm{~m})$. These findings corroborate those of a previous study of elderly subjects (Janyacharoen et al., 2013) also showing that Thai dance exercises for 6 weeks improve endurance and are in agreement with the present results.

Likewise, Eyigor et al. (2009) applied a Turkish folkloric dance-based exercise program 3 times per week for 8 weeks in women $73.5 \pm 7.6$ years old, while the control group $(71.2 \pm 5.5$ years old) did not have any exercise. Each Turkish folkloric dance session was divided into a 10-minute warm-up, 40 minutes of Turkish folklore dance, and 10 minutes of stretching and cool-down activities. Turkish folkloric dance routines included walking forward and backward, standing on one leg, semi-flexion of both knees and bouncing on knees rhythmically, moving the arms sideways while holding the little fingers of the participants, etc, performed in an order and accompanied by rhythm with all participants forming a circle. After the 8-week Turkish dances program, elderly women cardiorespiratory fitness, as measured by the 6MWT was significantly improved. More specifically, the 
travelled distance was increased from $419.1 \pm 84.1 \mathrm{~m}$ to $488.8 \pm 51.2 \mathrm{~m}$, while no significant difference was observed in the control group (Eyigor et al., 2009).

Shanahan et al. (2016) assessed aerobic fitness in regular participants in Irish dances $64 \pm 8$ years old, and agematched controls $69 \pm 14.5$ years old. Dancing group was attending weekly or biweekly set dancing classes in the 6-month period prior to the study, while the control group was not attending any dance class. Irish set dancing included repetitive multidirectional movement in group formation, different set dancing steps and in particular reel and polka steps with musical accompaniment. From the results, a significant difference was observed in the travelled distance in the 6MWT between dancers $(450 \pm 72.5 \mathrm{~m})$ and age-matched controls (395.5 $\pm 95 \mathrm{~m})$ (Shanahan et al., 2016).

Hui et al. (2009) applied a dancing program in women aged $68 \pm 4.5$ years. More specifically, the dancing exercise was specifically designed to meet the abilities of older persons. The movements were simplified and easy to learn, requiring the use of both upper and lower limbs, the back, extended arm movements and the involvement of major muscle groups. It consisted of cross steps and Cha-cha steps commonly found in group dancing, with the accompaniment of a well-known Canto-pop song played at a moderate pace. The duration of the intervention was 12 weeks, with a total of 23 sessions. Subjects in the dance group participated in two 50 min sessions per week, for 6 weeks initially, followed by two 60 -min sessions per week for another 6 weeks. For the first 6 weeks, each session consisted of 10 min of warming up, $30 \mathrm{~min}$ of dance with brief rests in between, followed by $10 \mathrm{~min}$ to cool down. For the following 6 weeks, the duration of dancing was increased from 30 to $40 \mathrm{~min}$. After the 12-week dancing program, Hui et al. (2009) demonstrated significant improvements in resting $\mathrm{HR}$ and exercise tolerance as was measured by the 6MWT. In detail, resting HR decreased from $74.00 \pm 11.00$ to $71.38 \pm 11.81 \mathrm{bpm}$ (change $-2.62 \pm 7.63 \mathrm{bpm}$ ) and cardiorespiratory fitness according to 6MWT increased from $412.5 \pm 62.30$ to $453.71 \pm 51.50 \mathrm{~m}$ (change $+41.21 \pm 39.38 \mathrm{~m}$ ).

The results of the impact of the dancing program of Hui et al. (2009) are comparable with the results of the present study, as was observed that resting HR decreased from $78.08 \pm 6.61$ to $74.23 \pm 5.18 \mathrm{bpm}$ (change -3.85 bpm) and cardiorespiratory fitness increased, according to 6MWT travelled distance from $570.15 \pm 40.26$ to $656.04 \pm 43.63 \mathrm{~m}$ (change $+85.89 \mathrm{~m}$ ). Of course the changes in the present study are bigger, both at resting HR and cardiorespiratory fitness, as the intensity of Hui's at al. (2009) dancing program is low, while the intensity of the present Greek traditional dancing program is moderate. In agreement, Adiputra et al. (1996) found that dancing lowered the resting HR and blood pressure and increased the maximum aerobic capacity in young males after an 8-week program of Balinese dance exercise.

The above mentioned dance types are social dance kinds and have many similarities with the Greek traditional dances. It is worth to be mentioned that although the above programs are based on different dancing modes led to similar results, concerning the cardiorespiratory fitness, as measured by the 6MWT and resting HR. These physical improvements observed may be due to the aerobic effects of dance. More specifically, Greek traditional dances, Turkish folkloric dances, Irish set dancing, Thai dance, dancing with cross steps and Cha-cha steps all embody aerobic exercise and socialization under the accompaniment of music. They include repetitive multidirectional movement, various standings on the feet, turning around, and co-ordinated arm movements on the rhythm of the accompanying music in cycle formation.

Thus, dancing predominantly involves contracting and exercising large muscles of the legs for certain duration. Hence, improvements in functional fitness tests involving dynamic movements of the lower limbs would be expected, such as in lower limb endurance and 6MWT (Hui et al., 2009). This complex integration of stimulatory input creates an enjoyable and energetic setting and may stimulate neuroplastic preservation of balance and physical fitness (Shanahan et al., 2016).

In addition, old people's participation in other forms of dance, such as Tai Chi, ballroom dance, social dance, lasting from 8-weeks to multiyear, led to beneficial effects on physical performance, functional capacity, balance, cognitive performance, motor function and quality of life compared to age-matched controls (Kattenstroth et al., 2011; Maciaszek, \& Osiński, 2010; Zhang et al., 2008). Moreover, low-impact dance 
programs, lasting 12-week, can improve cardiorespiratory fitness, and muscular strength and endurance in healthy older women (Hopkins et al., 1990; Shimamoto et al., 1998), and slow down deterioration of functional status in nursing home residents, as well (Machacova et al., 2015).

Similarly, aerobic exercise also increases the travelled distance in 6MWT in patients with ankylosing spondylitis and COPD (Borghi-Silva et al., 2009; Durmuş et al., 2009). So long as aerobic exercise also increases the distance travelled in 6MWT, the Greek traditional dances protocol used in the present study may be equivalent to an aerobic exercise protocol. In addition, it is worth to be mentioned that aerobic training, in the elderly, leads to favorable adaptations of the cardiovascular and respiratory system, similar to those observed in young people (Seals et al., 1984). Therefore, menopausal women should be encouraged to perform any aerobic exercise to improve their cardiorespiratory fitness. Otherwise, their functional capacity may decrease naturally. Besides, functional improvement is critical in the older people with regard to the ability to maintain daily activities independently (Eyigor et al., 2009).

On the other side, dance as a means of improving cardiorespiratory parameters is effective, as any other type of exercise (Rodrigues-Krause et al., 2016). For example, both aerobic dance and walking sessions are of adequate intensity to improve aerobic fitness in elderly people (Grant et al., 2002). Thus, dancing can produce physical results, such as physical fitness improvement, comparable with those of formal exercise training (Hwang, \& Braun, 2015). Given the accessibility and prominence of dance among older persons, and the fact that folkloric dance rhythms are more impressive and fun, especially for this age group, since they bear tunes special for the country (Eyigor et al., 2009), our findings indicate that Greek traditional dances can be developed as a readily available means for positive effects in health, functional capacity and quality of life promotion in postmenopausal women.

\section{Conclusions}

The present study provides evidence for the effectiveness of moderate impact aerobic dance in improving the cardiorespiratory fitness of a group of postmenopausal women in Greece. Given that dancing is a popular, famous and beloved activity, mainly among women, it could be said that Greek traditional dances may be an alternative exercise mode for postmenopausal women cardiorespiratory fitness improvement. In conclusion, programs with Greek traditional dances could be used for physical fitness improvement and maintenance.

\section{Conflicts of Interest}

The authors declare that there are no conflicts of interests.

\section{References}

1. Adiputra, N., Alex, P., Sutjana, D.P., Tirtayasa, K., Manuaba, A. (1996). Balinese dance exercises improve the maximum aerobic capacity. Journal of Human Ergology, 25: 25-29.

2. American Heart Association. (2011). Effectiveness-based guidelines for the prevention of cardiovascular disease in women-2011. Journal of the American College of Cardiology, 57(12): 1404-1423.

3. American Heart Association. (2014). Heart disease and stroke statistics. Update, A report from the American Heart Association. Circulation, 129: 28-292.

4. American Thoracic Society (2002). Statement (2002): Guidelines for the six-minute walk test. Am J Respir Crit Care Med, 166: 111-117.

5. Argiriadou, Eir. (2013). The examination of the effects of the participation in Greek dances in perceived health state of adults. Doctoral Dissertation, University of Peloponnesos, Department of Sports Organization \& Management, Greece (in Greek). 
6. Argiriadou, Eir., Mountakis, C., Konstadinakos, P., Zakas, A., Mavrovouniotis, F., Mavrovounioti, Chr. (2013). The effect of a single bout of Greek dances on subjective health of middle-aged people. Journal of Physical Education and Sport (JPES), 13(2): 177-183.

7. Argiriadou Eir., Mavrovouniotis F., Mavrovouniotis A., Mavrovounioti Ch., Nikitaras N., Mountakis C. (2017). Greek traditional dances program and self-evaluated effects and changes in life. World Journal of Research and Review, 5(6): 19-24.

8. Arts Council England (2006). Dance and Health, the benefits of people of all ages. www.artscouncil.org.uk/advice-and-guidance/browse-advice-and-guidance/dance-and-health-thebenefits-for-people-of-all-ages

9. Asikainen, T.M., Miilunpalo, S., Kukkonen-Harjula, K., Nenonen, A., Pasanen, M., Rinne, M., Uusi-Rasi, K., Oja, P., Vuori, I. (2003) Walking trials in postmenopausal women: effect of low doses of exercise and exercise fractionization on coronary risk factors. Scand J Med Sci Sports, 13: 284-292.

10. Barr, R.G., Wentowski, C.C., Grodstein, F., Somers, S.C., Stampfer, M.J., Schwartz, J., Speizer, F.E., Camargo, C.A.Jr. (2004). Prospective study of postmenopausal hormone use and newly diagnosed asthma and chronic obstructive pulmonary disease. Arch Intern Med, 164(4): 379-386.

11. Bird, S., Radermacher, H., Feldman, S., Sims, J., Kurowski, W., Browning, C., Thomas, S. (2009). Factors influencing the physical activity levels of older people from culturally-diverse communities: an Australian experience. Ageing and Society, 29(8): 1275-1294.

12. Blackman, L., Hunter, G., Hilyer, J., Harrison, P. (1988). The effects of dance team participation on female adolescent physical fitness and self-concept. Adolescence, 23: 437-448.

13. Blumenthal, J., Matthews, K., Fredrikson, M., Rifai, N., Schniebolk, S., German, D., Steege, J., Rodin, J. (1991). Effects of exercise training on cardiovascular function and plasma lipid, lipoprotein and apolipoprotein concentrations in premenopausal and postmenopausal women. Arteriosclerosis, Thrombosis \& Vascular Biology, 11(4): 912-917.

14. Borghi-Silva, A., Arena, R., Castello, V., Simões, R.P., Martins, L.E., Catai, A.M., Costa, D. (2009). Aerobic exercise training improves autonomic nervous control in patients with COPD. Respir Med, 103: 15031510.

15. Butland, R.J., Pang, J., Gross, E.R., Woodcock, A.A., Geddes, D.M. (1982). Two-, six-, and 12-minute walking tests in respiratory disease. Br Med J (Clin Res Ed), 284(6329): 1607-1608.

16. Denazi, E., Mavrovouniotis, F., Kouidi, E., Deligiannis, A., Argiriadou, Eir. (2013). The effect of a Greek traditional dances program on functional capacity in elderly women. Sport- und Präventivmedizin, 43(1): 6-11.

17. Diakoumakou, O., Diakoumakos, N. (2010). Menopause and lipid metabolism. Cardiological Opinion, 5(2): 79-83 (in Greek).

18. Durmuş, D., Alayli, G., Uzun, O., Tander, B., Cantürk, F., Bek, Y., Erkan, L. (2009). Effects of two exercise interventions on pulmonary functions in the patients with ankylosing spondylitis. Joint Bone Spine, 76: 150-155.

19. Edwards, B., Li, J. (2013). Endocrinology of menopause. Periodontology 2000, (61): 177-194. 
20. Elisaf, M., Pitsavos, Chr., Lymperopoulos, E., Giomalos, K., Athyros, V. (2014). Revised guidelines of the Hellenic Society for Atherosclerosis for the diagnosis and treatment of dyslipidaemias - 2014. Hellenic Review of Atherosclerosis, 5(3): 151-163 (in Greek).

21. Enright, P., Sherill, D. (1998). Reference equations for the Six-minute walk in healthy adults. Am J Respir Crit Care Med, 158: 1384-1387.

22. Eyigor, S., Karapolat, H., Durmaz, B., Ibisoglu, U., Cakir, S. (2009). A randomized controlled trial of Turkish folklore dance on the physical performance, balance, depression and quality of life in older women. Arch Gerontol Geriatr, 48: 84-88.

23. Farrel, S., Braun, L., Barlow, C., Cheng, Y., Blair, S. (2002). The relation of body mass index, cardiorespiratory fitness, and all-cause mortality in women. Obes Res, 10(6): 417-423.

24. Grant, S., Corbett, K., Todd, K., Davies, C., Aitchison, T., Mutrie, N., Henderson, B., Dargie, E. (2002). A comparison of physiological responses and rating of perceived exertion in two models of aerobic exercise in men and women over 50 years of age. British Journal of Sports Medicine, 36(4): 276-281.

25. Green, J., Stanforth, P., Rankinen, T., Leon, A., Rao, D., Skinner, J., Bouchard, C., Wilmore, J. (2004). The effects of exercise training on abdominal visceral fat, body composition, and indicators of the metabolic syndrome in postmenopausal women with and without estrogen replacement therapy. The HERITAGE Family Study Metabolism, 53(9): 1192-1196.

26. Haddock, B., Hopp, H., Mason, J., Blix, G., Blair, S. (1998). Cardiorespiratory fitness and cardiovascular disease risk factors in postmenopausal women. Medicine \& Science in Sports \& Exercise, 30(6): 893898.

27. Haddock, B., Marshak, H., Mason, J., Blix, G. (2000). The effect of hormone replacement therapy and exercise on cardiovascular disease risk factors in postmenopausal women. Sports Med Jan, 29(1): 39-49.

28. Hagner, W., Hagner-Derengowska, M., Wiacek, M., Zubrzycki, I. (2009). Changes in level of $\mathrm{VO}_{2 \mathrm{max}}$ blood lipids, and waist circumference in the response to moderate endurance training as a function of ovarian aging. Menopause: The Journal of The North American Menopause Society, 16(5): 1009-1013.

29. Hallal, P.C., Andersen, L.B., Bull, F.C., Guthold, R., Haskell, W., Ekelund, U. (2012). Global physical activity levels: surveillance progress, pitfalls, and prospects. The Lancet, 380 (9838): 247-257.

30. Halverstadt, A., Phares, D., Wilund, K., Goldberg, A., Hagberg, J. (2007). Endurance exercise training raises high-density lipoprotein cholesterol and lowers small low-density lipoprotein and very lowdensity lipoprotein independent of body fat phenotypes in older men and women. Metabolism Clinical \& Experimental, 56: 444-450.

31. Haskell, W.L., Blair, S.N., Hill, J.O. (2009). Physical activity: Health outcomes and importance for public health policy. Preventive Medicine, 49(4): 280-282.

32. Hopkins, D.R., Murrah, B., Hoeger, W.W., Rhodes, R.C. (1990). Effect of low-impact aerobic dance on the functional fitness of elderly women. The Gerontologist, 30: 189-192. doi:10.1093/geront/30.2.18

33. Hu, F.B., Willett, W.C., Li, T., Stampfer, M.J., Colditz, G.A., Manson, J.E. (2004). Adiposity as compared with physical activity in predicting mortality among women. The New England Journal of Medicine, 351: 2694-2703. 
34. Hui, E., Chui, B.T., Woo, J. (2009). Effects of dance on physical and psychological well-being in older persons. Arch Gerontol Geriatrics, 49(1): 45-50.

35. Hwang, P.W., Braun, K.L. (2015). The effectiveness of dance interventions to improve older adults' health: A systematic literature review. Altern Ther Health Med., 21(5): 64-70.

36. International Sport and Culture Association (2015). The economic cost of physical inactivity in Europe. Retrieved from: http://inactivity-time-bomb.nowwemove.com/download-report/The Economic Costs of Physical Inactivity in Europe (June 2015).pdf

37. Janyacharoen, T., Laophosri, M., Kanpittaya, J., Auvichayapat, P., Sawanyawisuth, K. (2013). Physical performance in recently aged adults after 6 weeks traditional Thai dance: a randomized controlled trial. Clin Interv Aging, 8: 855-859.

38. Janyacharoen, T., Phusririt, C., Angkapattamakul, S., Hurst, C.P., Sawanyawisuth, K. (2015). Cardiopulmonary effects of traditional Thai dance on menopausal women: a randomized controlled trial. J Phys Ther Sci, 27(8): 2569-2572.

39. Judge, J.A. (2003). Balance training to maintain mobility and prevent disability. American Journal of Preventive Medicine, 25(3 Suppl. 2): 150-156.

40. Karolkiewicz, J., Michalak, E., Pospieszna, B., Deskur-Smielecka, E., Nowak, A., Pilaczynska-Szczesniak, K. (2009). Response of oxidative stress markers and antioxidant parameters to an 8-week aerobic physical activity program in healthy, postmenopausal women. Archives of Gerontology \& Geriatrics, 49: 67-71.

41. Kattenstroth, J.C., Kalisch, T., Kolankowska, I., Dinse, H.R. (2011). Balance, sensorimotor, and cognitive performance in long-year expert senior ballroom dancers. J Aging Res, 10: e176709. doi: $10.4061 / 2011 / 176709$.

42. Kelley, G., Kelley, K., Tran, Z. (2004). Aerobic exercise and lipids and lipoproteins in women: a metaanalysis of randomized controlled trials. J Womens Health (Larchmt), 13(10): 1148-1164.

43. King, A. (2001). Interventions to promote physical activity by older adults. Journals of Gerontology, 56A(Special Issue II): 36-46.

44. King, A., Haskell, W., Young, D., Oka, R., Stefanick, M. (1995). Long-term effects of varying intensities and formats of physical activity on participation rates, fitness, and lipoproteins in men and women aged 50 to 65 years. Circulation, 91: 2596-2604.

45. King, M.B., Whipple, R.H., Gruman, C.A., Judge, J.O., Schmidt, J.A., Wolfson, L.I. (2002). The performance enhancement project: improving physical performance in older persons. Arch. Phys. Med. Rehabil., 83: 1060-1069.

46. Knopp, R. (2002). Risk factors for coronary artery disease in women. Am J Cardiol, 89: 28-35.

47. Kolovou, G., Bilianou, H. (2008). Influence of aging and menopause on lipids and lipoproteins in women. Angiology, 59(suppl 2): 54-57.

48. Korshoj, M., Lidegaard, M., Krustrup, P., Jorgensen, M.B., Sogaard, K., Holtermann, A. (2016). Long term effects on risk factors for cardiovascular disease after 12-months of aerobic exercise intervention - A worksite RCT among cleaners. PLOS ONE, 11(8): 1-22. Retrieved from http://dx.doi.org/10.1371/journal.pone.0158547 
49. Lindheim, S., Notelovitz, M., Feldman, E., Larsen, S., Khan, F., Lobo, R. (1994). The independent effects of exercise and estrogen on lipids and lipoproteins on postmenopausal women. Obstetrics \& Gynecology, 83: 167-172.

50. Lin, X., Zhang, X., Guo, J., Roberts, C.K., McKenzie, S., Wu, W.C., Liu, S., Song, Y. (2015). Effects of exercise training on cardiorespiratory fitness and biomarkers of cardiometabolic health: A systematic review and meta-analysis of randomized controlled trials. Journal of the American Heart Association, 4: 1-28. doi: 10.1161/JAHA.115.002014

51. Machacova, K., Vankova, H., Volicer, L., Veleta, P., Holmerova, I. (2015). Dance as prevention of late life functional decline among nursing home residents. Journal of Applied Gerontology: 1-18. doi: $10.1177 / 0733464815602111$.

52. Maciaszek, J., Osiński, W. (2010). The effects of tai chi on body balance in elderly people-a review of studies from the early 21st century. Am J Chin Med, 38: 219-229.

53. Makrilakis, K., Panagiotakos, D., Pitsavos, Chrysohoou, C., Ioannidis, I., Dimosthenopoulos, C., Toutouzas, P., Stefanadis, C., Katsilambros, N. (2004). The association between physical activity and the development of acute coronary syndromes in diabetic subjects (the CARDIO2000 II study). European Journal of Cardiovascular Prevention \& Rehabilitation, 11: 298-303.

54. Mavroudi, M., Mavroudi, E., Vavilis, T. (2010). Menopause and atherosclerosis Hellen Obstet Gynecol, 22(4): 143-147 (in Greek).

55. Mavrovouniotis, A., Argiriadou, Eir., Mavrovouniotis, F., Mavrovounioti, Chr., Mountakis, C., Nikitaras, N., Deligiannis, A. (2016). The evaluation of physical health of elderly participants or non-participants in a Greek dances program. Journal of Physical Education and Sport (JPES), 16 Supplement issue (1), Art 116: 713-719.

56. Mavrovouniotis, F., Argiriadou, Eir. (2008). Dance, old people and psychosomatic health. Inquiries in Sport \& Physical Education, 6(2): 222-231.

57. Mavrovouniotis, F., Argiriadou, Eir., Papaioannou, Chr. (2010). Greek traditional dances and quality of old people's life. Journal of Bodywork \& Movement Therapies, 14(3): 209-218.

58. Mensink, G., Deketh, M., Mul, M., Schuit, A., Hoffmeister, H. (1996). Physical activity and its association with cardiovascular risk factors and mortality. Epidemiology, 7(4): 391-397.

59. Mora, S., Cook, N., Buring, J., Ridker, P., Lee, I-M. (2007). Physical activity and reduced risk of cardiovascular events: Potential mediating mechanisms. Circulation, 116(19): 2110-2118.

60. North American Menopause Society, (2010). Estrogen and progestogen use in postmenopausal women: 2010 position statement of The North American Menopause Society. Menopause, 17(2): 242-255. doi: 10.1097/gme.0b013e3181d0f6b9.

61. Oguma, Y., Shinoda-Tagawa, T. (2004). Physical activity decreases cardiovascular disease risk in women: review and meta-analysis. American Journal of Preventive Medicine, 26(5): 407-418.

62. Palacios, S., Henderson, V., Siseles, N., Tan, D., Villaseca, P. (2010). Age of menopause and impact of climacteric symptoms by geographical region. Climacteric, 13: 419-428.

63. Papaioannou, Chr., Argiriadou, Eir., Mavrovouniotis, F. (2009/2010). The effect of Greek traditional dances on elderly women's well-being. Woman and Sports, 7: 25-38 (in Greek). 
64. Real, F.G., Svanes, C., Omenaas, E.R., Antò, J.M., Plana, E., Jarvis, D., Janson, C., Neukirch, F., Zemp, E., Dratva, J., Wjst, M., Svanes, K., Leynaert, B., Sunyer, J. (2008). Lung function, respiratory symptoms, and the menopausal transition. J Allergy Clin Immunol, 121: 72-80.

65. Rikli, R., Jones, C.J. (2012). Development and validation of criterion-referenced clinically relevant fitness standards for maintaining physical independence in later years. The Gerontologist, 53(2): 255-267.

66. Rodrigues-Krause, J., Farinha, J.B., Krause, M., Reischak-Oliveira, A. (2016). Effects of dance interventions on cardiovascular risk with ageing: Systematic review and meta-analysis. Complementary Therapies in Medicine, 29: 16-28. https://doi.org/10.1016/j.ctim.2016.09.004

67. Roussel, M., Garnier, S., Lemoine, S., Gaubert, I., Charbonnier, L., Auneau, G., Mauriege, P. (2009). Influence of a walking program on the metabolic risk profile of obese postmenopausal women. Menopause: The journal of the North American Menopause Society, 16(3): 566-575.

68. Rousseau, M.E. (1998). Women's Midlife Health Reframing Menopause. Journal of Nurse-Midwifery, 43(3): 208-223.

69. Seals, D., Hurley, B., Schultz, J., Hagberg, J. (1984). Endurance training in older men and women II. Blood lactate response to submaximal exercise. J Appl Physiol Respir Environ Exerc Physiol, 57(4): 1030-1033.

70. Se-Hong, K., Minjeong, K., Yu-Bae, A., Hyun-Kook, L., Sung-Goo, K., Jung-hyoun, C., Seo-Jin, P., SangWook, S. (2011). Effect of dance exercise on cognitive function in elderly patients with metabolic syndrome: A pilot study. J Sports Sci Med, 10(4): 671-678.

71. Shanahan, J., Coman, L., Ryan, F., Saunders, J., O'Sullivan, K., Bhriain, O.N., Clifford, A.M. (2016). To dance or not to dance? A comparison of balance, physical fitness and quality of life in older Irish set dancers and age-matched controls. Public Health, 141: 56-62, https://doi.org/10.1016/j.puhe.2016.07.015

72. Shimamoto, H., Adachi, Y., Takahashi, M., Tanaka, K. (1998). Low impact aerobic dance as a useful exercise mode for reducing body mass in mildly obese middle-aged women. Journal of Physiological Anthropology, 17: 109-114.

73. Solway, S., Brooks, D., Lacasse, Y., Thomas, S. (2001). A qualitative systematic overview of the measurement properties of functional walk tests used in the cardiorespiratory domain. Chest, 119: 256270.

74. Swisher, A., Goldfarb, A. (1998). Use of the Six-minute walk/run test to predict peak oxygen consumption in older adults. Cardiopulm Phys Ther J, 9: 3-5.

75. Thompson, P.D., Buchner, D., Pina, I.L., Balady, G.J., Williams, M.A., Marcus, B.H., Berra, K., Blair, S.N., Costa, F., Franklin, B., Fletcher, G.F., Gordon, N.F., Pate, R.R., Rodriguez, B.L., Yancey, A.K., Wenger, N.K. (2003). Exercise and physical activity in the prevention and treatment of atherosclerotic cardiovascular disease: A Statement from the Council on Clinical Cardiology. Circulation, 107: 3109-3116.

76. Vincent, K.R., Braith, R.W., Felman, R.A., Magyari, P.M., Cutler, R.B., Persin, S.A., Lennon, S.L., Gabr, A.H., Lowenthal, D.T. (2002). Resistance exercise and physical performance in adults aged 60 to 83 . J. Am. Geriatr. Soc., 50: 1100-1107.

77. Vryonidou, A., Klonaris, N. (2010). Menopause transition: pathophysiology, symptomatology and treatment. Proceedings of postgraduate courses, Intensive training in endocrinology: 13th cyclegonads, pp. 251-262. Retrieved from http://e-endocrinology.gr/wp-content/uploads/2014/05/1320.pdf (in Greek). 
78. WHO-World Health Organization (2010). Global recommendations on physical activity for health. Geneva, World Health Organization, 2010. Retrieved from: http://apps.who.int/iris/bitstream/10665/44399/1/9789241599979_eng.pdf

79. WHO-World Health Organization (2011). Global status report on noncommunicable diseases 2010. Geneva, World Health Organization, 2011. Retrieved from: http://www.who.int/nmh/publications/ncd_report2010/en/

80. WHO-World Health Organization (2014). Noncommunicable Diseases (NCD) Country Profiles, 2014. Retrieved from: http://www.who.int/nmh/publications/ncd-profiles-2014/en/

81. Zapantis, G., Santoro, N. (2003). The menopausal transition: characteristics and management. Best Practice \& Research Clinical Endocrinology \& Metabolism, 17(1): 33-52.

82. Zhang, J.G., Ishikawa-Takata, K., Yamazaki, H., Morita, T., Ohta, T. (2008). Postural stability and physical performance in social dancers. Gait Posture, 27: 697-701.

\section{About Fotios Mavrovouniotis}

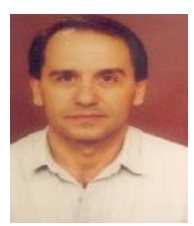

Fotios Mavrovouniotis is Associate Professor at the School of Physical Education and Sport Sciences, Aristotle University of Thessaloniki, GREECE 